A-09-2020

\title{
EFECTO DEL MANEJO DEL RIEGO SOBRE LA PRODUCCIÓN Y CALIDAD POSTCOSECHA DE NECTARINA
}

\author{
Conesa M.R., Conejero, W., Vera, J., Ruiz-Sánchez, M.C.(P)
}

Departamento de Riego. CEBAS-CSIC. Campus Universitario de Espinardo, Apartado 164, 30100, Murcia, España

mrconesa@cebas.csic.es; wenceslao@cebas.csic.es; jvera@cebas.csic.es; mcruiz@cebas.csic.es

\section{Resumen}

En este trabajo se evalúan los efectos de diferentes condiciones de manejo del riego sobre la producción, calidad y conservación de nectarina extratemprana. Se aplicaron 3 tratamientos: dos con programación del riego en base a $100 \% \mathrm{ETc}$, con un lateral de riego ( $\mathrm{T}$-Simple) y dos laterales (T-Doble), y programación del riego automática (T-Automático) en base a sensores de agua en el suelo. A pesar de la doble superficie de suelo humectada en T-Doble se observó un déficit ligero durante la postcosecha (disminución de potencial hídrico de tallo a mediodía $\left(\Psi_{\mathrm{t}, \mathrm{md}}\right)$ de $0,15 \mathrm{MPa}$ respecto a T-Simple), que no afectó de forma significativa a la producción y calidad de la nectarina. La programación automática en base a sensores generó un déficit moderado, con diferencias de $\Psi_{t, m d}$ de $0,25 \mathrm{MPa}$ respecto a TSimple durante la postcosecha. T-Automático supuso un ahorro de agua del $35 \%$, sin afectar a los parámetros de producción (salvo un aumento del contenido en sólidos solubles totales, SST) y mejorando la eficiencia del uso del agua, EUA (32\% > T-Simple). Durante el periodo de conservación (10 días a $0{ }^{\circ} \mathrm{C}+5$ días a $15^{\circ} \mathrm{C}$ ), las nectarinas del T-Automático mostraron los valores más altos de SST y del índice de madurez, mientras que las del TDoble mostraron los valores más altos de luminosidad (L). Los frutos de todos los tratamientos se evaluaron sensorialmente como aptos para la comercialización, no obstante, los del T-Automático fueron los mejor clasificados por el consumidor.

\section{1) Introducción}

Las variedades de maduración extra-temprana dan su nombre a la pronta recolección, que para el caso de la nectarina en condiciones mediterráneas acontece entre finales de abril y principios de mayo. En estos meses, el clima es menos extremo, lo que permite reducir el consumo de agua sin mermar el rendimiento productivo (Ruiz-Sánchez et al., 2018). No obstante, los frutos cosechados se caracterizan por poseer bajo contenido en sólidos solubles (SST $<10{ }^{\circ}$ Brix) y elevada acidez titulable. Además, atributos como el sabor, la apariencia visual o la textura están afectados por las condiciones ambientales y el aporte hídrico (Génard et al., 2007).

Investigaciones recientes, han mostrado un incremento en la calidad del fruto del nectarino mediante la aplicación de riego deficitario durante la postcosecha y/o antes de la recolección (Falagán et al., 2015; López et al., 2016). Sin embargo, no existen muchos estudios sobre la respuesta productiva y de calidad de la nectarina a diferentes condiciones de programación del riego.

El diseño agronómico del sistema de riego por goteo más común en cultivos frutales es el de un único lateral porta-emisores por hilera de árboles (Conesa et al., 2019). La hipótesis planteada es que el mismo volumen de agua aplicado en dos líneas portaemisores, favorecería un mayor volumen humectado, así como la retención y difusión de la humedad del suelo, reduciendo la acumulación de sales, la evapotranspiración, y, en 
consecuencia, mejorando la eficiencia del riego y la productividad del agua. Además, la integración de las nuevas tecnologías en los sistemas de riego por goteo, como el uso de sensores de agua en el suelo y su gestión telemática, contribuyen a la toma de decisiones de cuándo, cómo regar y qué cantidad aplicar de forma automática (Vera et al., 2019).

\section{2) Objetivos}

Los objetivos del trabajo fueron: (i) evaluar la respuesta productiva de nectarinos extra-tempranos al manejo del riego con programación basada en ETc (con uno y dos laterales de riego) y riego automatizado basado en sensores de agua del suelo, y (ii) evaluar la calidad postcosecha de la nectarina en las distintas condiciones de manejo del riego.

\section{3) Materiales y Métodos}

\section{1.) Condiciones de la parcela experimental}

El ensayo tuvo lugar durante el ciclo de crecimiento 2018-2019 en una parcela de $\approx$ 0,5 ha de la finca experimental del CEBAS-CSIC, situada en Santomera-Murcia $\left(38^{\circ} 06^{\prime}\right.$ ' N, $1^{\circ} 02^{\prime}$ O), de nectarinos extra-tempranos (Prunus persica (L.) Batsch cv. 'Flariba'), de 8 años de edad, injertados sobre GxN-15, y distribuidos a un marco de plantación de 6,5 m x 3,5 m. El suelo era calizo, pedregoso y poco profundo, con una textura franco-arcillosa, una densidad aparente de $1,43 \mathrm{~g} \mathrm{~cm}^{-3}$ y bajos niveles de materia orgánica $(1.3 \%)$ y capacidad de intercambio catiónico $\left(97,9 \mathrm{mmol} \mathrm{kg}^{-1}\right)$. El agua de riego procedente del trasvase TajoSegura presentó valores medios anuales de conductividad eléctrica $\left(C_{25^{\circ} \mathrm{C}}\right)$ de $1,3 \mathrm{dS} \mathrm{m}^{-1} \mathrm{y}$ $\mathrm{pH}$ cercano a 8. El contenido de agua en el suelo $(\Theta v)$ a capacidad de campo (CC) y punto de marchitez (PM) fue del 29 y $14 \%$, respectivamente. Los árboles recibieron 83-56-109 kg ha ${ }^{-1}$ y año-1 de $\mathrm{N}, \mathrm{P}_{2} \mathrm{O}_{5}$ y $\mathrm{K}_{2} \mathrm{O}$, respectivamente, mediante fertirriego (Vera y de la Peña, 1994). Las prácticas culturales (control de malas hierbas, tratamientos fitosanitarios, aclareo, etc.) fueron las típicas para frutales de hueso de la zona.

\section{2.) Tratamientos de manejo del riego}

Se aplicaron 3 tratamientos de riego: (i) T-Simple: regado al $100 \%$ de la evapotranspiración del cultivo $\left(E T_{c}\right)$ con una línea porta-goteros, con 4 goteros de $4 \mathrm{~L} \mathrm{~h}^{-1}$ dispuestos a 50 y $130 \mathrm{~cm}$ del tronco; (ii) T-Doble: regado al $100 \% \mathrm{ET}_{\mathrm{c}}$ con dos líneas portagoteros, separadas entre sí $1 \mathrm{~m}$, con 8 goteros de $4 \mathrm{~L} \mathrm{~h}^{-1}$ y dispuestos 50 y $130 \mathrm{~cm}$ del eje del tronco; y (iii) T-Automático: regado según valores umbrales de $\Theta_{\vee}$ monitorizados con sondas de capacitancia, con el mismo sistema de riego que T-Simple.

En T-Automático, los valores de $\Theta_{v}$ fueron establecidos de acuerdo a umbrales de déficit hídrico $(\alpha)$ del $10 \%$ durante el crecimiento del fruto y la postcosecha inicial, y del $30 \%$ durante la postcosecha final, calculados respecto al agotamiento de agua máximo permitido (MAD) (Vera et al., 2019). El riego se activaba de manera automática cuando los valores de $\Theta_{v}$ alcanzaban el MAD, y cesaba cuando alcanzaban el umbral de CC. El valor de a se incrementó durante la postcosecha final para evitar un excesivo crecimiento vegetativo del árbol.

La $\mathrm{ET}_{\mathrm{c}}$ fue estimada como el producto de la evapotranspiración de referencia $\left(\mathrm{ET}_{0}\right.$, según ecuación de Penman-Monteith, Allen et al., 1998) y coeficientes de cultivo locales (Kc, Abrisqueta et al., 2013). El diseño experimental consistió en 4 repeticiones distribuidas al azar de 6 árboles por repetición, utilizándose los 4 centrales para las medidas.

\section{3.) Medidas realizadas durante la precosecha}

El $\Theta_{v}$ fue monitoreado en continuo con 4 sondas de capacitancia EnviroScan $®$ (Sentek Sensor Technologies, Adelaida, Australia) por tratamiento de riego (1 sonda por repetición), previamente calibradas (Evett et al., 2006; Abrisqueta et al., 2012). Los tubos de 
acceso de PVC fueron instalados a $10 \mathrm{~cm}$ del emisor situado a $50 \mathrm{~cm}$ del tronco. Cada sonda de capacitancia tenía sensores a 10, 30, 50 y $70 \mathrm{~cm}$ de profundidad que estaban conectados de forma inalámbrica a una unidad de transmisión de radio. Los sensores leen cada 5 min y la media de 15 min es enviada a través de un sistema de telemetría (ADCON Telemetry Smart Solutions, Austria). La visualización y procesamiento de los datos se realizó en la plataforma web addVANTAGE versión pro 6.6. Más detalles sobre el sistema de riego automatizado pueden consultarse en Vera et al. (2019).

El estado hídrico de la planta fue evaluado con medida del potencial hídrico de tallo a mediodía $\left(\Psi_{\mathrm{t}, \mathrm{md}}\right)$ con cámara de presión (Soil Moisture Equipment Corp., Model 3000 ) en 4 hojas sanas por tratamiento de riego (1 hoja por repetición) situadas en la zona Norte y cerca del tronco de los árboles, las cuales fueron cubiertas con papel de aluminio $2 \mathrm{~h}$ antes de la medida, siguiendo las recomendaciones de Hsiao (1990).

La sección transversal del tronco (STT) se determinó con pie de rey (CodimexC100cm, Canadá) a $30 \mathrm{~cm}$ del suelo en 4 árboles por repetición y tratamiento y el diámetro ecuatorial del fruto con calibre digital (Mitutoyo, CD-15D) en 20 frutos por repetición y tratamiento.

En la cosecha (realizada la primera semana de mayo) se controló el peso total, número de frutos por árbol y \% de frutos agrietados, en todos los árboles del ensayo $(n=24$ por tratamiento). La eficiencia productiva (EP) fue estimada como el cociente entre la producción total y la STT. La eficiencia de uso del agua (EUA) fue determinada como el cociente entre la producción total y el volumen de riego aplicado en cada tratamiento.

\section{4.) Ensayo de conservación postcosecha. Medidas de calidad física, química y sensorial.}

Tras la cosecha, unos $20 \mathrm{~kg}$ de nectarinas por tratamiento se transportaron a la cámara de almacenamiento frigorífico situada en las instalaciones del CEBAS-CSIC. Allí se seleccionaron 45 frutos sanos y sin defectos por cada repetición ( $n=180$ frutos por tratamiento): 15 frutos/repetición se dedicaron al estudio de calidad físico-química de la cosecha y el resto se dispusieron en cajas con alveolos que fueron almacenados durante 10 días a $0^{\circ} \mathrm{C}$ y humedad relativa (HR) del $90-95 \%$ (periodo de almacenamiento en frío) y 5 días a $15^{\circ} \mathrm{C}$ y $\mathrm{HR}=70-75 \%$ (periodo de simulación de vida comercial).

Al final de cada periodo: día 0 (cosecha), día 10 (fin de almacenamiento en frío) y día 15 (fin de simulación de vida comercial) se hicieron las siguientes determinaciones:

\section{- Parámetros de calidad física.}

En 15 frutos por repetición (60 por tratamiento) se realizaron las siguientes medidas:

- Pérdida de peso: con balanza de precisión (Mettler PE 360 DeltaRange, Suiza).

- Firmeza: con texturómetro (AGROSTA® 100Field, Francia).

- Color: con colorímetro (Minolta CR-10, Osaka, Japón) según el sistema de coordenados CIE L*a*b*. A partir de estos valores, se calculó el croma [C* $\left(a^{*} 2+b^{*} 2\right) 1 / 2$ ] y el tono o matiz [ángulo hue, $h^{\circ}=\operatorname{ta}^{n-1}\left(b^{*} / a^{*}\right)$ ].

\section{- Parámetros de calidad química.}

Se utilizaron 5 frutos por repetición (20 por tratamiento) que fueron licuados y en el jugo obtenido se realizaron las siguientes medidas:

- Sólidos solubles totales (SST) expresados en ${ }^{\circ}$ Brix: con medidor digital (Atago PAL-BX/ACID F5 Master Kit- Multifrutas, Japón).

- Acidez titulable (AT): con medidor digital (Atago PAL-BXIACID F5 Master KitMulti-frutas, Japón). Los valores obtenidos fueron expresados en mg ácido málico $\mathrm{L}^{-1}$ jugo de nectarina.

- Índice de Madurez (IM): Estimado como el cociente SST/ AT. 
- pH y CE: Con medidor Cyberscan (Model PCD-6500, Nijkerk, Holanda) a temperatura ambiente.

\section{- Análisis sensorial}

Al final de cada periodo de conservación (días 10 y 15 del experimento) un panel de 10 personas ( 6 mujeres y 4 hombres, entre 25 y 60 años) realizaron una cata sensorial que mostró las preferencias del consumidor, siguiendo las indicaciones de Falagán et al. (2015) con algunas modificaciones. Entre otros, se evaluó la apariencia visual, sabor, textura y la calificación global, para lo que se utilizó una escala hedónica de 5 puntos siendo: 1: extremadamente malo / textura muy blanda; 2 : me disgusta / textura blanda; 3 : ni me gusta ni disgusta / moderada textura (límite de comercialización); 4, me gusta / buena textura y 5 : me encanta / excelente textura.

\section{5.) Análisis estadístico.}

A los datos obtenidos se aplicó un análisis de varianza (ANOVA) utilizando el programa SPSS versión 13.0 para Windows. Para los del ensayo de conservación postcosecha se consideró un modelo bifactorial (tratamiento de manejo del riego y tiempo de conservación, como factores principales) y las medias se compararon con el test de la mínima diferencia significativa (MDS) a un nivel de confianza del 95\% (MDS ${ }_{0.05}$ ).

\section{4) Resultados y Discusión.}

Durante el periodo de estudio, la cantidad de agua de riego aplicada en T-Simple y TDoble fue de 470 y $449,2 \mathrm{~mm}$, respectivamente, mientras que el tratamiento T-Automático supuso una reducción en el riego del 35\% respecto al T-Simple. Esta reducción (considerando una tarifa de este período de $0,35 € / \mathrm{m}^{3}$ según datos de la Comunidad de Regantes con fecha 1 de febrero de 2019) supondría un ahorro anual en los costes de agua de $\approx 584 €$. Nótese que para el tratamiento T-Simple, el coste sería de unos $1645 €$.

Durante el periodo de estudio se registró una precipitación de $392 \mathrm{~mm}$, distribuida de forma irregular como es característico de la zona (Fernández-García et al., 2020). La evapotranspiración de referencia $\left(E_{0}\right)$ fue de $1081 \mathrm{~mm}$, con valores máximos de 6,98 mm día ${ }^{-1}$ en los meses más cálidos y de $0,36 \mathrm{~mm}$ día $^{-1}$ en los meses más fríos, lo que supone fuertes déficits hídricos estacionales (Fig. 1A).

Los valores de $\Theta_{v}$ en el tratamiento T-Simple se mantuvieron próximos a capacidad de campo $(29 \%)$ a lo largo de la estación, mientras que en planta los valores de $\Psi_{\mathrm{t}, \mathrm{md}}$ oscilaron entre $-0,4$ y $-0,7 \mathrm{MPa}$, correspondientes a condiciones hídricas no limitantes (Girona et al., 2005; Vera et al., 2013, 2019) (Fig. 1B-D).

A pesar de la mayor superficie humectada, el riego con doble lateral indujo un estrés leve durante la postcosecha, con una disminución de $\Psi_{\mathrm{t}, \mathrm{md}}$ de $0,15 \mathrm{MPa}$ y $\Theta_{\mathrm{v}} 11 \%$ más bajo que T-Simple. Girona et al. (2005) y Naor et al. (2005) establecieron un valor umbral de $\Psi_{\mathrm{t}, \mathrm{md}}$ de -1,5 MPa para garantizar la fertilidad y floración del melocotonero y de -2,0 MPa para limitar la aparición de frutos dobles. Por su parte, la programación del riego en T-Automático supuso una reducción media en los valores de $\Theta_{v}$ del $13 \%$ respecto a T-Simple, que se tradujeron en niveles de déficit hídrico moderado en planta durante la postcosecha final, con una disminución de $\Psi_{\mathrm{t} \text {,md }}$ de $0,25 \mathrm{MPa}$ respecto a T-Simple y un valor mínimo de $-1,7 \mathrm{MPa}$ en octubre (Fig. 1B-D). En cuanto al diámetro del fruto, no se observaron diferencias significativas entre tratamientos (Fig. 1C). López et al., (2016) indicaron que un ligero déficit $\left(\Psi_{\mathrm{t}, \mathrm{md}} \approx-1,25 \mathrm{MPa}\right)$ aplicado antes de la recolección puede afectar negativamente al tamaño y producción final del nectarino. 

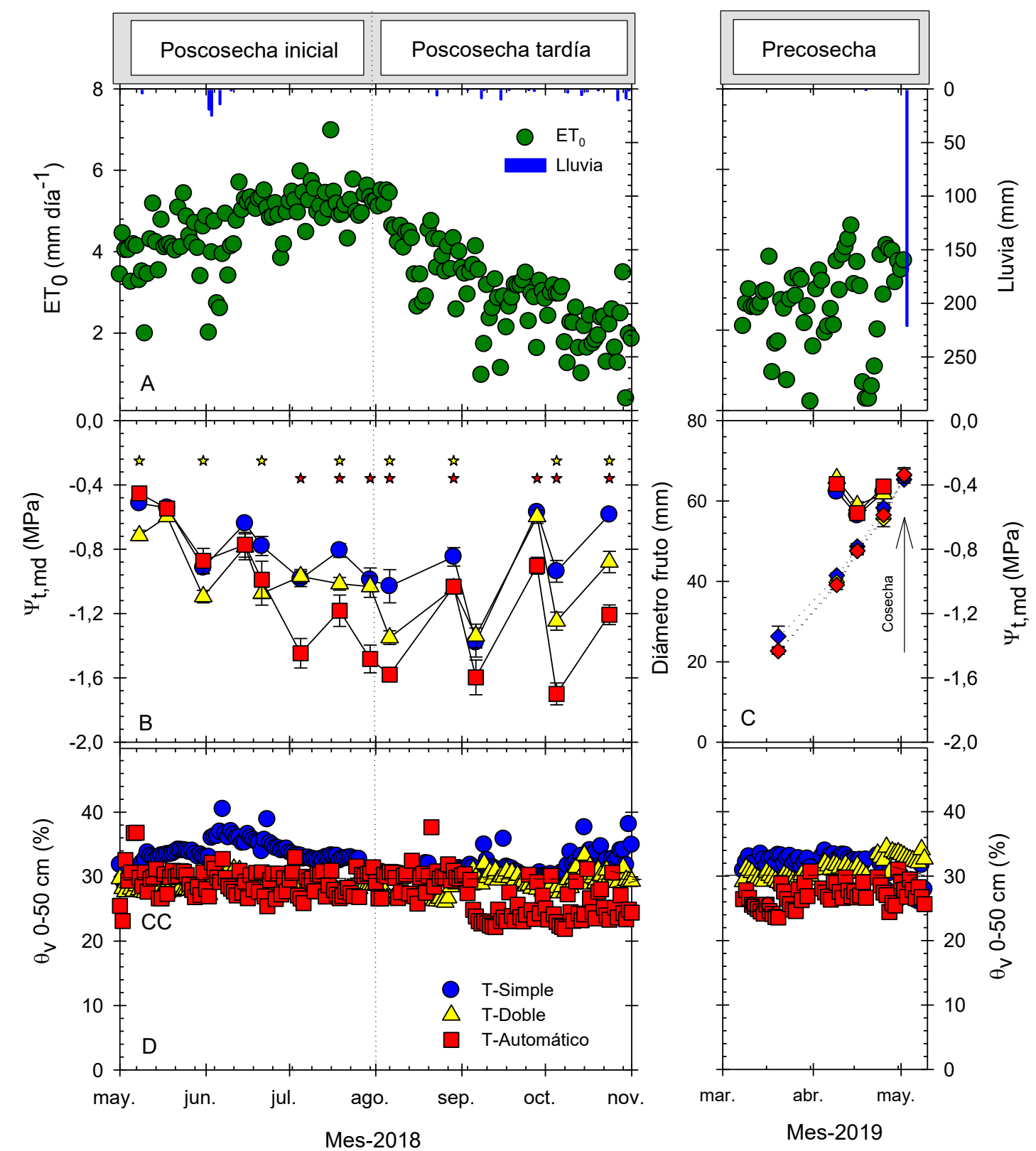

Figura 1. Evolución estacional de: (A) Evapotranspiración de referencia $\left(E T_{0}, \mathrm{~mm} \mathrm{día}^{-1}\right)$ y lluvia ( $\left.\mathrm{mm}_{\text {día }}{ }^{-1}\right)$; (B) Potencial hídrico de tallo a mediodía $\left(\Psi_{\mathrm{t}, \mathrm{md}}, \mathrm{MPa}\right)$; (C) Diámetro ecuatorial del fruto ( $\mathrm{mm}, \diamond)$; y (D) Humedad volumétrica del suelo en el perfil $0-50 \mathrm{~cm}\left(\Theta_{v}, \%\right)$ en los tratamientos T-Simple $\left({ }^{-}\right)$, T-Doble $(-\triangle)$ ) y T-Automático $(\rightarrow-)$ ). Cada punto es la media de 4 hojas, 80 frutos y 12 sensores \pm error estándar (en D se omite para mejor visualización). Línea horizontal en $\mathrm{D}=$ capacidad de campo. Los asteriscos indican diferencias significativas respecto a T-Simple según el test de la MDS $_{0.05}$.

No se observaron diferencias significativas entre tratamientos, en ninguno de los parámetros productivos estudiados (producción total y $\mathrm{n}^{\circ}$ de frutos por árbol, peso medio de fruto y $\%$ de frutos agrietados), si bien la eficiencia de uso del agua (EUA) fue significativamente mayor en el tratamiento T-Automático, con un incremento del $32 \%$ respecto a T-Simple (Tabla 1). Es importante señalar que los valores de producción total se refieren solo a frutos de calidad comercial. Conesa et al. (2019) indicaron que la programación del riego con sondas de capacitancia permitió un ahorro del agua del $40 \%$ sin producir mermas en la producción final del nectarino extratemprano en relación con la programación de riego tradicional basada en ETc. 
La sección trasversal del tronco y la eficiencia productiva fueron similares en los tratamientos de manejo del riego (Tabla 1). Millán et al. (2019) observó en ciruelo que la programación de riego deficitario de forma automática en base a sensores de humedad del suelo tuvo resultados similares de rendimiento y vigor de planta a los de riego deficitario convencional basado en porcentajes de reducción de la ETc.

Tabla 1. Parámetros productivos de nectarinos en los tratamientos de manejo del riego.

\begin{tabular}{lccc}
\hline & T-Simple & T-Doble & T-Automático \\
\cline { 2 - 4 } Producción $\left(\mathrm{kg} \mathrm{árbol}^{-1}\right)$ & 23,73 & 25,32 & $22,75 \mathrm{~ns}$ \\
Carga productiva $\left(\mathrm{n}^{\circ}\right.$ frutos árbol $\left.{ }^{-1}\right)$ & 166,5 & 180,5 & $154,75 \mathrm{~ns}$ \\
Peso medio fruto $(\mathrm{g})$ & 150,07 & 149,96 & $152,9 \mathrm{~ns}$ \\
Frutos rajados $(\%)$ & 45,01 & 32,82 & $37,57 \mathrm{~ns}$ \\
Sección trasversal del tronco $\left(\mathrm{STT}, \mathrm{cm}^{2}\right)$ & 269,5 & 207,4 & $220,4 \mathrm{~ns}$ \\
Eficiencia productiva $\left(\mathrm{EP}, \mathrm{kg} \mathrm{cm}^{-2}\right)$ & 0,08 & 0,12 & $0,10 \mathrm{~ns}$ \\
Eficiencia uso del agua $\left(\mathrm{EUA}, \mathrm{kg} \mathrm{m}^{-3}\right)$ & $5,04 \mathrm{a}$ & $5,63 \mathrm{a}$ & $7,50 \mathrm{~b}$ \\
\hline
\end{tabular}

ns: no significativo. Letras distintas indican diferencias significativas según el test de MDS $_{0.05}$

El moderado déficit hídrico generado en T-Automático incrementó los SST ( $\left.{ }^{\circ} B r i x\right)$ en la cosecha (Tabla 2), con valores cercanos al óptimo de consumo (Crisosto et al., 1994). Esto puede ser debido a una síntesis más activa de ácidos orgánicos en respuesta al estrés hídrico y como mecanismo de ajuste osmótico (Pedrero et al., 2014). Durante el periodo de conservación (10 días a $0{ }^{\circ} \mathrm{C}+5$ días a $15^{\circ} \mathrm{C}$ ), las nectarinas del T-Automático mostraron los valores más altos de SST ( ${ }^{\circ} \mathrm{Brix}$ ) e IM, el cual estuvo motivado por un descenso en AT, mientras que las del T-Doble registraron los valores más altos de luminosidad (L). El descenso de AT tras la comercialización, también apreciada en melocotón bien regado y con RDC (Gelly et al., 2004), indica un consumo de ácidos orgánicos como sustrato respiratorio del fruto. El incremento de SST en frutos sometidos a RDC ha sido observado en nectarino (Falagán et al., 2015; López et al., 2016), ciruelo (Intringliolo y Castel et al., 2010), granado (Laribi et al., 2013), melocotonero (Rahmati et al., 2014) y peral (López et al., 2011). En general, dicho incremento se relaciona con una mejora en el sabor de la fruta que implica una mayor aceptación por parte del consumidor, quien percibe un sabor más dulce (Crisosto et al., 1994). Sin embargo, y a pesar del incremento de ${ }^{\circ}$ Brix en T-Automático, el panel de cata sensorial no reflejó esa sensación debido al carácter astringente de la variedad de nectarina. En este sentido, López et al. (2016) indicó que los consumidores apreciaron cambios en la calidad de nectarinas sometidas a estrés hídrico, que no correspondieron con los resultados obtenidos por instrumentos objetivos de medida (e.j. refractómetro, colorímetro), por lo que proponen la calidad sensorial del consumidor como una variable sensible para el análisis de programas de riego deficitario. De forma general, el tiempo de conservación ( $\mathrm{t}$ ), afectó de forma más significativa a la calidad físico-química de la nectarina, que el tratamiento de riego $(\mathrm{R})$ (Tabla 2).

La pérdida de peso de los frutos fue también similar e inferior al $7 \%$ en todas las nectarinas al final del ensayo de conservación (Fig. 2), hecho que puede achacarse a la formación de una cutícula más gruesa como mecanismo de defensa al estrés hídrico, que previene la deshidratación de los frutos durante el periodo de conservación (Falagán et al., 2014). Además, Mpelasoka et al. (2001) señalaron una menor incidencia de ataques fúngicos debido a la formación de dicha cutícula. Otros autores han indicado menores pérdidas de peso en albaricoques conservados a $1^{\circ} \mathrm{C}$ durante 30 días (Pérez-Pastor et al., 2007). La apariencia visual y textura (o ablandamiento) de los frutos fue significativamente mayor en T-Automático respecto a T-Doble y T-Simple (Fig. 3A, B). La calidad sensorial global de los frutos fue apta para el consumo en todos los tratamientos (Fig. 3D). 
Tabla 2. Calidad físico-química de las nectarinas en cosecha (día 0), fin periodo de almacenamiento en frío (día 10) y fin periodo de vida comercial (día 15) en los tratamientos de manejo del riego.

\begin{tabular}{|c|c|c|c|c|c|c|c|c|c|c|}
\hline & $\begin{array}{c}\text { Diámetro } \\
\text { ecuatorial } \\
(\mathrm{mm})\end{array}$ & $\begin{array}{c}\text { Firmeza } \\
\text { (\%) }\end{array}$ & $L^{*}$ & ${ }^{\circ} \mathrm{Hue}$ & $\begin{array}{l}\text { Croma } \\
\left({ }^{\circ} \mathrm{C}\right)\end{array}$ & $\begin{array}{c}\text { SST } \\
\text { ('Brix) }\end{array}$ & $\begin{array}{c}\text { AT } \\
\left(\mathrm{mg} \mathrm{L}^{-1}\right)\end{array}$ & IM & CE & $\mathrm{pH}$ \\
\hline \multicolumn{11}{|l|}{ día 0} \\
\hline T-Simple & 65,33 & 88,6 & 38,38 & 57,97 & 27,97 & 9,57 a & 13,42 & 7,77 & 4,5 & $3,5 a b$ \\
\hline T-Doble & 66,37 & 86,8 & 39,7 & 57,98 & 29,73 & 9,82 a & 14,62 & 7,58 & 4,8 & $3,46 \mathrm{a}$ \\
\hline T-Automático & 66,53 & 86,5 & 38,52 & 57,96 & 28,09 & 10,72 b & 10,65 & 10,17 & 4,68 & $3,57 b$ \\
\hline \multicolumn{11}{|l|}{ día 10} \\
\hline T-Simple & 65,83 & 88,77 & $37,11 \mathrm{a}$ & 57,95 & 29,18 & $9,8 a b$ & 10,12 & 9,7 & 4,5 & 3,55 \\
\hline T-Doble & 67,21 & 89,27 & $38,94 \mathrm{~b}$ & 57,96 & 31,26 & 9,65 a & 9,72 & 9,92 & 4,41 & 3,61 \\
\hline T-Automático & 65,69 & 89,42 & $38,03 a b$ & 57,96 & 29,29 & 10,62 b & 9,97 & 10,64 & 4,4 & 3,46 \\
\hline \multicolumn{11}{|l|}{ día 15} \\
\hline T-Simple & 64,98 & 49,45 & $38,15 a$ & 57,93 & 31,56 & 10,07 & $14,77 a b$ & $7,04 a b$ & 3,76 a & 3,95 \\
\hline T-Doble & 65,63 & 49,6 & $40,48 \mathrm{~b}$ & 57,95 & 34,11 & 10,07 & $16,4 \mathrm{~b}$ & $6,2 \mathrm{a}$ & $3,96 \mathrm{a}$ & 3,87 \\
\hline T-Automático & 64,9 & 48,25 & $39,04 \mathrm{ab}$ & 57,92 & 32,46 & 10,25 & $11,32 \mathrm{a}$ & $9,67 \mathrm{~b}$ & $4,65 \mathrm{~b}$ & 3,89 \\
\hline \multicolumn{11}{|l|}{ ANOVA / Medias } \\
\hline Tratamiento riego $(R)$ & ns & ns & $* *$ & ns & ns & * & ns & * & ns & ns \\
\hline T-Simple & 65,48 & 75,6 & $37,71 \mathrm{ab}$ & 57,95 & 29,19 & 9,86 a & 12,92 & $8,2 \mathrm{a}$ & 4,24 & 3,68 \\
\hline T-Doble & 66,81 & 74,72 & $39,46 \mathrm{~b}$ & 57,96 & 31,4 & 9,85 a & 13,63 & 7,97 a & 4,34 & 3,64 \\
\hline T-Automático & 66,02 & 75,23 & $38,56 a$ & 57,95 & 29,96 & $10,53 b$ & 10,6 & $10,22 b$ & 4,58 & 3,66 \\
\hline Tiempo conservación (t) & ns & $* * *$ & ns & * & $* * *$ & ns & $*$ & * & $*$ & $* * *$ \\
\hline día 0 & 66,89 & $87,31 \mathrm{~b}$ & 38,48 & 57,97 b & 27,93 a & 10,10 & $13,04 \mathrm{~b}$ & $8,67 \mathrm{~b}$ & $4,64 \mathrm{~b}$ & $3,53 \mathrm{a}$ \\
\hline $\begin{array}{l}\text { día } 10 \\
\text { día } 15\end{array}$ & $\begin{array}{l}66,25 \\
65,18\end{array}$ & $\begin{array}{c}89,15 \mathrm{c} \\
49,1 \mathrm{a}\end{array}$ & $\begin{array}{l}38,03 \\
39,23\end{array}$ & $\begin{array}{l}57,96 \mathrm{~b} \\
57,93 \mathrm{a}\end{array}$ & $\begin{array}{l}29,91 \mathrm{~b} \\
32,71 \mathrm{c}\end{array}$ & $\begin{array}{l}10,03 \\
10,13\end{array}$ & $\begin{array}{c}9,94 \mathrm{a} \\
14,16 \mathrm{~b}\end{array}$ & $\begin{array}{c}10,09 b \\
7,63 a\end{array}$ & $\begin{array}{c}4,44 a b \\
4,12 a\end{array}$ & $\begin{array}{l}3,54 \mathrm{a} \\
3,91 \mathrm{~b}\end{array}$ \\
\hline Interacción $R \times t$ & ns & ns & ns & ns & ns & ns & ns & ns & ns & ns \\
\hline
\end{tabular}

Letras distintas indican diferencias significativas para la $\mathrm{MDS}_{0.05 .}{ }^{*} \mathrm{P}<0,05 ;{ }^{* *} \mathrm{P}<0,01 ;{ }^{* * *} \mathrm{P}<0,001$. ns :no significativo. 


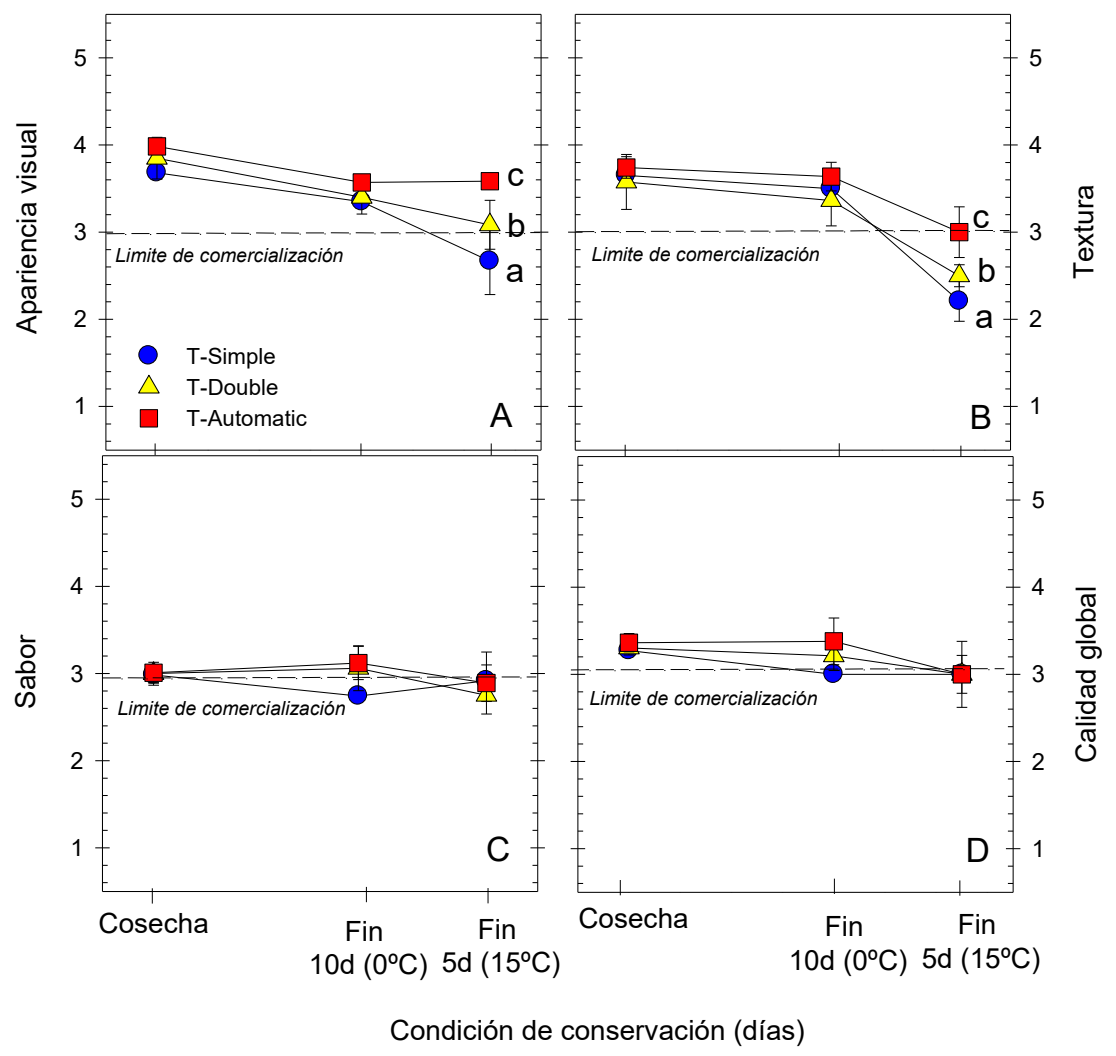

Figura 2. Apariencia visual (A), textura (B), sabor (C) y calidad global (D) de las nectarinas en la cosecha, al final del periodo de almacenamiento en frío y del periodo de simulación de vida comercial en los tratamientos T-Simple $\left({ }^{-}\right)$, T-Doble $(-\triangle-)$, y T-Automático $(-\square)$.

Los valores son media de 10 panelistas \pm error estándar. Letras diferentes indican diferencias significativas según el test de la $\mathrm{MDS}_{0.05}$.

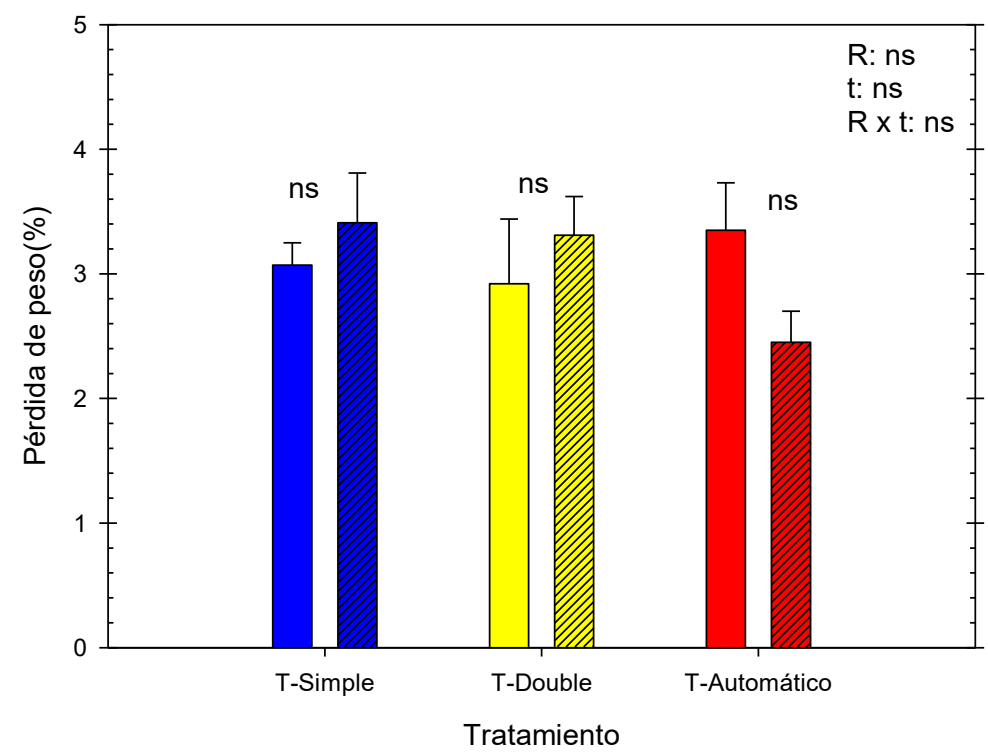

Figura 3. Pérdida de peso de las nectarinas al final del periodo de almacenamiento en frío (barras rellenas) y del periodo de simulación de vida comercial (barras rayadas) en los distintos tratamientos de manejo del riego. Los valores son medía de 4 repeticiones de 15 frutos ( $n=60$ frutos por tratamiento) \pm error estándar. ns: no significativo. ANOVA: Tratamiento riego $(\mathrm{R})$, tiempo conservación $(\mathrm{t})$, interacción $\mathrm{R} x \mathrm{t}$. 


\section{5) Conclusiones y Recomendaciones}

La programación del riego con sensores de agua en el suelo en el tratamiento TAutomático ha permitido ahorros importantes de agua de riego $(=35 \%)$, frente al riego basado en ETc, sin producir mermas en el rendimiento, calidad y vida postcosecha (conservación) de la nectarina, lo que incrementó la eficiencia de uso del agua en un 32\% respecto a T-Simple. El empleo de criterios agronómicos precisos junto al uso de dispositivos tecnológicos es una alternativa que mejorará la programación del riego, contribuyendo a la sostenibilidad de la agricultura. El riego con doble lateral no ha supuesto ninguna ventaja en términos de producción y calidad respecto al riego con lateral simple.

\section{6) Agradecimientos}

El trabajo se ha financiado con los proyectos AGL2016-77282-C03-1R y PID2019106226RB-C2-1 (Plan Nacional AEI-Fondos FEDER-UE) y 19903/GERM/15 (Fundación Séneca, Región de Murcia). M.R. Conesa agradece la ayuda posdoctoral disfrutada del programa Juan de la Cierva (FJCl-2017-32045).

\section{7) Bibliografía}

Abrisqueta, I., Abrisqueta, J., Tapia, L., Munguía, J., Conejero, W., Vera, J., RuizSánchez, M.C. (2013). Basal crop coefficients for early-season peach trees. Agricultural Water Management. 121, 158-163.

Abrisqueta, I., Vera, J., Tapia, L., Abrisqueta, J., Ruiz-Sánchez, M. (2012). Soil water content criteria for peach trees water stress detection during the postharvest period. Agricultural Water Management. 104, 62-67.

Allen, R.G., Pereira, L.S., Raes, D., Smith, M. (1998). Crop Evapotranspiration: Guidelines for Computing Crop Water Requirements; Food and Agriculture Organization of the United Nations: Rome, Italy.

Conesa, M.R., Conejero, W., Vera, J., Ramírez-Cuesta, J.M., Ruiz-Sánchez, M.C. (2019). Terrestrial and remote indexes to assess moderate deficit irrigation in early-maturing nectarine trees. Agronomy. 9 (10), e630.

Crisosto, C.H., Johnson, R.S., Luza, J.G., Crisosto, G.M. (1994). Irrigation regimes affect fruit soluble solids concentration and rate of water loss of 'O'Henry' peaches. HortScience. 29, 1169-1171.

Evett, S.R., Tolk, J.A., Howell, T.A. (2006). Soil profile water content determination. Vadose Zone J. 5, 894.

Falagán, N., Artés, F., Artés-Hernández, F., Gómez, P.A., Pérez-Pastor, A., Aguayo, W. (2015). Comparative study on postharvest performance of nectarines grown under regulated deficit irrigation. Postharvest Biology and Technology. 110, 24-32.

Falagán, N., Artés, F., Gómez, P.A., Artés-Hernández, F., Conejero, W., Aguayo, W. (2014). Deficit irrigation strategies combine with controlled atmosphere preserve quality in early peach. Food Science and Technology International. 21 (7), 547-556.

Fernández-García, I., Lecina, S., Ruiz-Sánchez, M.C., Vera, J., Conejero, W., Conesa, M.R., Dominguez A., Pardo, J.J., Léllis, B.C., Montesinos, P. (2020). Trends and challenges in irrigation scheduling in the semi-arid area of Spain. Water. 12 (3), e785.

Génard, M., Bertín, N., Borel,C., Bussiéres, P., Gautier, H., Habib, R. Léchaudel, M., Lecomte, A., Lescourret, F., Lobit, P., Quilot, B. (2007). Towards a virtual fruit focusing on quality: modelling features and potential uses. Journal of Experimental Botany. 58, 917-928.

Gelly, M., Recasens, I., Girona, J., Mata, M., Arbones, A., Rufat, A. y Marsal, J. (2004). Effects of stage II and postharvest deficit irrigation on peach quality during maturation and after cold storage. Journal of the Science of Food and Agriculture. 84, 561-568. 
Girona, J., Gelly, M., Mata, M., Arbonés, A., Rufat, J., Marsal, J. (2005). Peach tree response to single and combined deficit irrigation regimes in deep soils. Agricultural Water Management. 72, 97-108.

Hsiao, T.C. (1990). Measurement of tree water status. In: Steward, B,. A., Nielsen, D.R. (Eds), Irrigation of Agricultural Crops. Agronomy Monograph No.30. American Society of Agronomy. Madison. WI. pp 243-279.

Intrigliolo, D.S., Castel, J.R. (2010). Response of plum trees to deficit irrigation under two crop levels: tree growth, yield and fruit quality. Irrigation Science, 28, 525-534.

Laribi, A.I., Palou, L., Intrigliolo, D.S., Nortes, P.A., Rojas-Argudo, C., Taberner, V., Bartual, J., Pérez-Gago, M.B. (2013). Effect of sustained and deficit irrigation on fruit quality of pomegranate cv. 'Mollar de Elche' at harvest and during cold storage. Agricultural Water Management. 125, 61-70.

López, G., Larrigaudiere, C., Girona, J., Behboudian, M.H., Marsal J. (2011). Fruit thinning in 'conference' pear grown under deficit irrigation: implications for fruit quality at harvest and after cold storage. Scientia Horticulturae. 129 (1), 64-70.

López, G., Echeverria, G., Bellvert, J., Mata, M., Behboudian, M.H., Girona, J., Marsal, J. (2016). Water stress for a short period before harvest in nectarine: Yield, fruit composition, sensory quality, and consumer acceptance of fruit. Scientia Horticulturae. 211, $1-7$

Millán, S., Casadesús, J., Campillo, C., Moñino, M.J., Prieto, M.H. (2019). Using soil moisture sensors for automated irrigation scheduling in a plum crop. Water. 11, e2061.

Mpelasoka, B.S., Behboudian, M.H.,Mill, T.M. (2001). Effects of deficit irrigation on fruit maturity and quality of 'Braebourn' apple. Scientia Horticulturae. 90, 279-290.

Naor, A., Stern, R., Peres, M., Greenblat, Y., Gal, Y., Flaishman, M.A. (2005). Timing and severity of postharvest water stress affect following-year productivity and fruit quality of field-grown 'Snow Queen' nectarine. Journal of the American Society for Horticultural Science 130, 806-812.

Pedrero, F., Maestre-Valero, J.F., Mounzer, O.; Alarcón, J.J., Nicolás, E. (2014). Physiological and agronomic mandarin trees perfomance under saline reclaimed water combined with regulated deficit irrigation. Agricultural Water Management. 146, 228-237.

Pérez-Pastor, A., Ruiz-Sánchez, M.C., Martínez, J.A., Nortes, P.A., Artés, F. y Domingo, R. (2007). Effect of deficit irrigation on apricot fruit quality at harvest and during storage. Journal of the Science of Food and Agriculture. 87, 2409-2415.

Rahmati, M., Vercambre, G., Davarynejad, G.H., Bannayan, M., Azizi, M., Génard, M. (2014). Water scarcity conditions affect peach fruit size and polyphenol contents more severely than other fruit quality traits. Journal of the Science of Food and Agriculture. 95, 1055-1065.

Ruiz-Sánchez, M.C., Abrisqueta, I., Conejero, W., Vera, J. (2018). Deficit irrigation management in early-maturing peach crop. In: Water Scarcity and Sustainable Agriculture in Semiarid Environment. Tools, Strategies, and Challenges for Woody Crops. ISBN 978-0-12813164-0. Elsevier. Chapter 6. pp 111-126.

Vera, J., Abrisqueta, I., Abrisqueta, J.M., Ruiz-Sánchez M.C. (2013). Effect of deficit irrigation on early-maturing peach tree performance. Irrigation Science. 31, 747-757.

Vera, J., de la Peña, J.M. (1994). FERTIGA: Programa de Fertirrigación de Frutales; CEBAS-CSIC: Murcia, Spain, pp. 69.

Vera, J., Conejero, W., Conesa, M.R., Ruiz-Sánchez, M.C. (2019). Irrigation factor approach based on soil water content: A nectarine orchard case study. Water (11), e589. 